\title{
Yitang Zhang Awarded Rolf Schock Prize
}

YITANG ZHANG of the University of New Hampshire has been awarded the Rolf Schock Prize in mathematics for 2014 "for his spectacular breakthrough concerning the possibility of an infinite number of twin primes."

In April 2013, Zhang, a relatively unknown lecturer at the University of New Hampshire, stunned the mathematical world with an article on one of the oldest unsolved problems in mathematics, the so-called "twin primes conjecture".

Prime numbers do not seem to appear randomly; rather, they exhibit several different regularities. Nevertheless, it is not possible to specify clear rules for where in the whole number sequence prime numbers will appear. It is known, however, that the further along the number sequence, the longer the distance between the primes-they become ever more rare. Do they ever stop appearing? The answer is no, and was given 2,300 years ago by Euclid of Alexandria, who proved that there are indeed infinitely many prime numbers.

A puzzling characteristic of prime numbers is that they sometimes appear in pairs, following each other. When separated only by 2 , they are called twin primes. Are there an infinite number of such pairs? The proof of this so-called "twin prime conjecture" has been elusive. No one has yet been able to provide a definite answer, even though the question has engaged many well-known experts in analytical number theory. Yitang Zhang brought mathematics one important step closer to the answer. He managed to show that there are an infinite number of prime pairs for which the distance between the pair is less than 70 million. Zhang worked alone on the problem for many years, using an innovative approach, building on and developing previous work in various subfields of mathematics.
His results were groundbreaking and spurred great activity around the world. In a few months, a team of mathematicians, led by Terence Tao of the University of California, Los Angeles, managed to shrink the distance between the prime numbers from 70 million to 4,680 . A young postdoc at the University of Montreal, James Maynard, developed entirely new ideas and has now joined the collective endeavor. Together, they have reached the lowest limit of 270 between the primes in a pair. It remains uncertain, however, whether this distance can be reduced all the way down to 2 .

Yitang Zhang was born in China in 1955 and studied mathematics at the University of Beijing. He moved to the USA in 1985 and defended his doctoral thesis at Purdue University in 1992. After that, he held various casual jobs until 1999, when he joined the University of New Hampshire, where he recently was appointed Professor of Mathematics. He was awarded the 2014 Cole Prize in Number Theory by the AMS, as well as the 2013 Ostrowski Prize.

The Rolf Schock Prizes are awarded every three years in the fields of logic and philosophy, mathematics, the visual arts, and the musical arts. The prize amount is 600,000 Swedish kronas, approximately US\$93,000. They are awarded by the Royal Swedish Academy of Sciences, the Royal Swedish Academy of Fine Arts, and the Royal Swedish Academy of Music. - From a Royal Swedish Academy
of Sciences news release 